\title{
Tangence
}

\section{Les écrivains... et les « auteurs jeunesse »}

\section{Edith Madore}

Numéro 67, automne 2001

L'écriture pour la jeunesse : de la production à la réception

URI : https://id.erudit.org/iderudit/009613ar

DOI : https://doi.org/10.7202/009613ar

Aller au sommaire du numéro

Éditeur(s)

Presses de l'Université du Québec

ISSN

0226-9554 (imprimé)

1710-0305 (numérique)

Découvrir la revue

\section{Citer cet article}

Madore, E. (2001). Les écrivains... et les « auteurs jeunesse ». Tangence, (67), 23-33. https://doi.org/10.7202/009613ar
Résumé de l'article

Résumé

Quelle est la place des écrivains - ceux qui écrivent pour différents publics, celui des jeunes et celui des adultes - et celle des «auteurs jeunesse» - ceux qui écrivent uniquement pour les jeunes dans le(s) champ(s) littéraire(s)? Les auteurs jeunesse n'en- trent pas impunément dans le circuit de la littérature générale. Les écrivains pour adultes n'entrent pas non plus comme ils le veulent dans la sphère jeunesse. Cependant, certains éditeurs jeunesse tendent maintenant à publier aussi pour les adultes, et les deux sphères semblent s'interpénétrer. 


\section{Les écrivains... et les « auteurs jeunesse» Édith Madore, Université Laval}

D'entrée de jeu, précisons que ce titre, volontairement provocateur, a été emprunté à Denis Côté, un "auteur jeunesse", qui séparait ainsi ironiquement les écrivains pour adultes de ceux qui écrivent pour les jeunes. De fait, l'appellation "auteurs jeunesse" est fréquemment utilisée par les professionnels du milieu du livre, ce qui agace plus d'un écrivain pour les jeunes. Cet agacement est un symptôme qui vient révéler un malaise plus profond, que notre recherche entend prendre pour objet: quelle est la place des écrivains - ceux qui écrivent pour différents publics, celui des jeunes et celui des adultes - et celle des "auteurs jeunesse" - ceux qui écrivent uniquement pour les jeunes - dans le(s) champ(s) littéraire(s)?

Plusieurs éléments de cette analyse prennent appui sur ma thèse de doctorat ${ }^{1}$, laquelle interrogeait des questions relatives à la sociologie de la littêrature et à la problématique des champs littéraires, suivant une perspective qui est celle de Bourdieu et de Viala. J'aborderai les deux points suivants: les trajectoires d'écrivains - pour montrer quels écrivains occupent une position dominante dans le milieu littéraire et quels sont le statut et la définition de l'aauteur jeunesse" - et l'autonomisation du champ, dans le but d'observer la lutte pour la légitimité dans le(s) champ(s) littéraire(s). Je m'appuierai enfin sur les travaux de Dominique Noguez portant sur la définition de l'écrivain. Je crois en effet que la notion de grantécrivain introduite par Noguez peut servir à établir un parallèle avantageux entre l'écrivain et l' auteur jeunesse", de manière à penser ces deux types d'écrivains à partir d'un point de vue unique et par-delà les différences présumées de statut qui existeraient entre eux.

\section{Les trajectoires d'écrivains pour la jeunesse}

La trajectoire exemplaire empruntée par les écrivains pour la jeunesse se fonde sur l'obtention de prix littéraires. Elle épouse le

1. Édith Madore, Constitution de la littérature québécoise pour la jeunesse, 1920-1995, thèse de doctorat, Université Laval, Québec, 1996. 
parcours suivant: publication, critique, obtention d'un prix littéraire. L'écrivain qui remporte de nombreux prix bénéficie d'une plus grande visibilité, et son impact sur le champ littéraire s'accrôt.

Qui détient le prestige? Quels écrivains, issus de quelles catégories, occupent une position dominante dans le milieu littéraire? Les organismes de promotion de la littérature québécoise pour la jeunesse ont contribué à définir l'écrivain et les différentes catégories au sein desquelles peuvent se regrouper les différentes trajectoires qu'ils empruntent. C'est un phénomène analogue qui, du reste, avait intéressé Alain Viala à propos de la littérature d'Ancien Régime, alors qu'il y observait à quel point

Les conflits qui parcourent ce monde se manifestent en particulier dans les palmarès des "bons auteurs". Ceux-ci valent comme prises de positions en face de ces clivages: la sélection qu'ils opèrent et les critères qu'ils utilisent isolent parmi l'ensemble des auteurs quelques catégories présentées comme les modèles de l'art du bien-écrire. Or le nom d'"écrivain" y devient de plus en plus le critère décisif, et ceux qui sont ainsi qualifiés forment de plus en plus un milieu. La plupart de ces palmarès sont des catalogues ou des séries d'appréciations critiques? 2 .

Entre la pratique des lettres au xvIr siècle et la littérature québécoise pour la jeunesse qui a cours de nos jours, doit-on s'étonner si une sociologie littéraire peut sans trop de peine retrouver à l'ouvre les mêmes mécanismes de légitimation? En effet, quelle que soit la nature des publications, au palmarès des "bons auteurs" n'apparaissent pas que les seules figures consacrées d'une époque. Aux côtés des écrivains que sanctionnent fortement l'école ou les prix littéraires et qui entrent automatiquement dans ces sélections - celles de Communication Jeunesse, par exemple, dans le Québec contemporain -, on retrouve aussi des auteurs qui, tout en empruntant une trajectoire obéissant davantage à la mode - songeons au roman socioréaliste -, entreront aussi dans le classement, même si ces écrivains ont peu publié ou n'ont pas ou peu remporté de prix littéraires.

Au fur et à mesure que se constitue le champ littéraire, le statut et la définition de l'écrivain pour la jeunesse se modifient. Comme le dit Pierre Bourdieu, "comprendre les pratiques des écrivains et des artistes, à commencer par leurs productions, c'est

2. Alain Viala, Naissance de lécrivain. Sociologie de la littérature à l'âge classique, Paris, Minuit, coll. "Le sens commun ", 1985, p. 245-246. 
comprendre qu'elles sont la résultante de la rencontre de deux histoires, l'histoire de la position, du poste qu'ils occupent, et l'histoire de leurs dispositions ${ }^{3}$. C'est ainsi qu'à partir des années 1980, l'équilibre devient plus précaire pour conserver sa position à cause des oppositions synchroniques entre des positions antagonistes (dominant/dominé, consacré/novice, etc.). Pierre Bourdieu affirme que "l'initiative du changement revient presque par définition aux nouveaux-entrants ${ }^{4}$. Ceux-ci veulent faire reconnaître leur différence en imposant de nouveaux modes d'expression, et ils sont dès lors en rupture avec les modes de pensée en vigueur. En 1982, par exemple, rien n'était moins à la mode que d'écrire des romans pour adolescents: les éditeurs interrogés affirmaient à qui voulait les entendre qu'il ne s'agissait pas là d'un bon marché, puisque les adolescents, prétendaient-ils, ne lisaient plus. Lorsque Raymond Plante, nouvellement arrivé sur le marché littéraire jeunesse au cours des années 1970 , crée une collection de romans destinés aux préadolescents et dans laquelle il fait paraître quelques-uns de ses ouvrages, il prend l'initiative d'un changement. Il parvient graduellement à affirmer son identité, puis, en 1986, survient un coup d'éclat: la publication de son roman pour adolescents, Le dernier des raisins. Des modes de pensée et d'expression nouveaux (récit de la vie quotidienne, sexualité, etc.), en rupture avec les modes de pensée en vigueur jusque-là, s'étaient frayés un chemin pendant ces années jusqu'à ce que cet auteur qui les représente obtienne la consécration suprême: le prix du Conseil des Arts du Canada en 1986 ${ }^{5}$. Autre exemple: Bertrand Gauthier, auteur et éditeur (jusqu'en 2001) de La courte échelle, revendique sa position dans le marché en affirmant que sa maison a été la première au Québec, en 1975, à produire des livres pour les jeunes. Cet éditeur déplore l'entrée de nouveaux venus dans le champ littéraire, car il prétend qu'ils n'apportent rien de plus au marché, bien au contraire ${ }^{6}$.

3. Pierre Bourdieu, "Le champ littéraire. Préalables critiques et principes de méthode", Lendemains, Berlin, n* 36, 1984, p. 15.

4. Pierre Bourdieu, "Le champ littéraire", art. cité, p. 24.

5. Depuis 1987, cette distinction est connue sous le nom de prix du Gouverneur général du Canada.

6. "Le MAC (ministère des Affaires culturelles) devrait aider davantage les éditeurs qui peuvent investir une meilleure pénétration du marché; car les autres produisent mais ne réussissent pas à vendre", dans Édith Madore, "Les éditeurs agréés ", Lurelu, Montréal, vol. 15, n” 3, hiver 1993, p. 40. 
De leur côté, les petits éditeurs, nouvellement arrivés, affirment qu'ils sont les principaux agents de l'innovation, tâche qu'ils ne sauraient néanmoins mener à bien sans que les revues et les organismes octroyant des subventions ne leur accordent un appui plus marqué qu'aux gros éditeurs déjà bien implantés depuis plusieurs années. Bref, pour réclamer la place qui leur revient, ils font appel à la nouveauté et à leur capacité d'innover. Puisque "c'est déjà exister dans un champ que d'y produire des effets, fûtce de simples réactions, de résistance ou d'exclusion ${ }^{7}$, on peut dire, au demeurant, que les dominants accordent l'existence à ceux qu'ils veulent exclure du seul fait qu'ils les combattent. La condamnation est une forme de reconnaissance en soi honorable car, dès qu'un éditeur suscite des attaques de la part des défenseurs de tel type d'édition, on peut dire qu'il existe. Ses détracteurs hâtent même sa reconnaissance.

Les écrivains engagent des luttes ou déploient des stratégies littéraires pour défendre ou améliorer leur position. Ces stratégies dépendent de la place de chacun au sein de rapports de force où l'on doit se démarquer pour exister. Le texte original marque un écart par rapport aux positions traditionnelles du champ et, dès qu'une ouvre parvient à occuper une position distincte, reconnaissable, l'oeuvre reconnue situe les autres et détermine l'évolution de leur valeur.

Les luttes de définition qu'entreprennent les écrivains pour conserver une position avantageuse déterminent le statut de l'écrivain. Chacun vise à imposer au champ littéraire les limites les plus favorables à ses intérêts ou à faire triompher sa définition des conditions de l'appartenance véritable au champ. Par exemple, les "purs", ou les agents du champ restreint, vont considérer volontiers qu'un certain nombre d'écrivains ne sont pas des artistes véritables, leur refusant l'existence en tant qu'artistes ${ }^{8}$ et en leur imposant du coup leurs propres critères. Ces "meurtres symboliques", ainsi Bourdieu les qualifie-t-il, visent à refuser l'existence en tant que véritables écrivains à des producteurs qui se disent écrivains au nom d'une définition plus large de la profession:

Un des enjeux centraux des luttes littéraires est le monopole de la légitimité littéraire, c'est-à-dire le monopole du pouvoir de

7. Pierre Bourdieu, "Le champ littéraire", art. cité, p. 15.

8. Pierre Bourdieu, "Le champ littérairı", art. cité, p. 13. 
dire avec autorité qui est autorisé à se dire écrivain [...] ou, si l'on préfère, le monopole du pouvoir de consécration des producteurs ou des produits?.

On peut identifier les luttes qui visent à définir le statut de l'écrivain pour la jeunesse, et le rôle hégémonique qu'elles jouent, autant dans les revues littéraires que dans la presse quotidienne. Le Bulletin de l'Association des écrivaines et des écrivains québécois pour la jeunesse (AEQJ) peut nous renseigner sur quelques-unes de ces querelles, qu'il s'agisse du refus des modes passagères ou encore des présumés conflits d'intérêt des critiques-auteurs. L'AEQJ ne peut passer sous silence "une situation qui commence à créer un sérieux malaise dans [notre] milieu: les articles de ceux et celles qui sont à la fois critiques et auteurs. Nos collègues, Henriette Major et Robert Soulières, ont récemment souligné le problème ${ }^{10}$ ". En 1992, en effet, Henriette Major soulève la question dans la revue Lurelu $^{11}$ et, dans le numéro suivant, Dominique Demers, écrivaine et journaliste, affiche son désaccord ${ }^{12}$.

La multiplicité des agents conduit à des luttes de définition pour contrôler le principe de légitimité. Comme exemple de nomos - principe de vision et de division -, l'Union des écrivaines et écrivains québécois (UNEQ) a fait connaître, en 1994, un projet destiné à déterminer les critères d'appartenance à cet organisme voué à la défense des droits des écrivains: ce projet vise à distinguer l'écrivain (il écrit de la littérature) de celui qui ne l'est pas (il est un essayiste universitaire, il est un auteur de livres pratiques, etc.). L'entreprise témoigne à l'évidence de l'extrême diversité qui prévaut dès lors qu'il s’agit de définir postes ou fonctions; les principes de légitimité qui s'affrontent sont aussi nombreux que les catégories de producteurs (parents, éducateurs, écrivains, etc.). Outre les querelles d'appartenance qui sont relatives à ces diverses catégories, la tâche des producteurs se complexifie car, peu importe la catégorie à laquelle ils appartiennent,

9. Pierre Bourdieu, "Le champ littéraire", art. cité, p. 14.

10. Anonyme, "On est si peu, soyons grands!", Le Bulletin, Montréal, Association des écrivaines et des écrivains québécois pour la jeunesse (AEQJ), $\mathbf{n}^{\circ} \mathbf{1}$, septembre 1992, p. 2.

11. Henriette Major, "Quelques réflexions sur les auteurs-critiques", Lurelu, Montréal, vol. $15, n^{\circ} 1$, printemps-été 1992 , p. 34.

12. Dominique Demers, "Ne tirez pas trop vite sur les critiques", Lureh, Montréal, vol. 15, $\mathrm{n}^{\prime 2}$, automne 1992 , p. 50 . 
ceux-ci occupent une double position: ils jouent aussi le rôle de médiateurs auprès des jeunes, choisissant les œuvres qui leur conviennent le mieux.

Qui est en droit de participer à la lutte pour la définition de l'écrivain? Qui doit ou peut écrire pour les enfants? Des éducateurs? Des parents? Des "écrivains véritables ", c'est-à-dire des écrivains ayant déjà une carrière derrière eux? Depuis les débuts de la littérature pour la jeunesse, on constate que les écrivains qui cumulent déjà les honneurs dans l'ensemble du champ littéraire sont avantagés dans le champ littéraire jeunesse.

\section{L'autonomisation du champ}

Il n'y a aucune commune mesure entre la quasi-disparition de la littérature pour la jeunesse au début des années 1970 et la situation qui règne au début des années 1980. Stabilisation et régularité de la production, qualité et diversification des genres sont les mots qui qualifient le mieux ce changement spectaculaire causé par l'expansion éditoriale des années 1980. En très peu d'années, le livre jeunesse est rapidement devenu rentable.

Plusieurs facteurs expliquent ce revirement favorable: la mobilisation du milieu littéraire pour la jeunesse, les nouvelles politiques du livre et l'aide gouvernementale à l'édition, entre autres. Si les prix littéraires les plus importants sont créés à partir des années 1970 , il ne faut pas oublier que ce n'est qu'à compter des années 1950, sauf erreur, que les œuvres littéraires francophones pour les jeunes ont pu être récompensées par des prix spécifiques. En 1980, d'après des enquêtes de lecture ${ }^{13}$, les jeunes Québécois lisaient surtout des auteurs américains. Depuis 1993, on remarque le phénomène inverse: depuis lors, les auteurs québécois sont lus massivement. On peut donc parler de reconnaissance de la littérature pour la jeunesse par le public cible.

Les années 1970 constituent une période de restructuration du sous-champ littéraire québécois pour la jeunesse. La transition est telle que ce sous-champ échappe à la domination de l'ensem-

13. Hélène Sarrasin (rédaction), Maurice Dalois, Guy Legault et Hélène Sarrasin (recherche et analyse), La lecture chez les jeunes au secondaire, des policiers aux classiques, Gouvernement du Quêbec, ministère de lYíducation du Quĉbec, 1994 . 
ble du champ littéraire pour accéder à une sorte d'autonomie. On peut maintenant le qualifier de "champ littéraire québécois pour la jeunesse" car, depuis les années 1980, période capitale pour son évolution, la littérature pour la jeunesse s'est vraiment constituée en champ autonome.

Depuis les années 1980, période où le public a été gagné à cette littérature et où la question du champ national s'est estompée, le plus grave handicap de la littérature québécoise pour la jeunesse reste cependant la lutte pour la légitimité au sein du champ littéraire. C'est que la littérature pour la jeunesse, en grandissant, entre en conflit avec l'ensemble du champ littéraire. Un écrivain pour la jeunesse est-il un "vrai" écrivain? Un livre pour la jeunesse est-il un "vrai " livre? Le statut inférieur qu'accordent aux écrivains jeunesse journalistes et chercheurs laisse planer le doute: de fait, les œuvres jeunesse n'entrent pas dans l'histoire littéraire générale et une place moindre leur est accordée dans les journaux.

Cette marginalisation semble reposer principalement sur la question du public cible. En effet, un écrivain qui écrit pour les adultes, même s'il écrit des romans jeunesse, figure dans les dictionnaires d'auteurs et dans les manuels: c'est le cas d'Yves Beauchemin, par exemple. Cependant, un "auteur jeunesse" qui n'écrit que pour les enfants n'y sera pas mentionné, comme le montre l'exemple de Robert Soulières ou celui de Ginette Anfousse. Selon le public auquel il s'adresse, l'écrivain ne semble donc pas occuper la même place dans le(s) champ(s). En effet, dans les grands quotidiens, à la radio et à la télévision, la littérature pour la jeunesse n'est pas représentée de façon proportionnelle à celle des adultes. Par ailleurs, dans le but d'élargir leur couverture médiatique et de se procurer ainsi des honneurs réservés traditionnellement aux auteurs pour adultes, certains éditeurs - de plus en plus nombreux - décident de publier une trilogie, ou deux romans jeunesse reliés ensemble, sous la couverture d'un volume destiné aux adultes. C'est le cas, notamment, de la trilogie de Dominique Demers, publiée aux éditions Québec/Amérique (Marie Tempête), et d'un volume double de Benjamin Simard, publié aux éditions Michel Quintin (Ben Caribou). Est-il bien certain que l'on veuille offrir à ces "auteurs jeunesse" une plus grande audience? Ne voudrait-on pas plutôt que ces derniers soient davantage pris au sérieux par tous les agents du champ littéraire? comme l'affirme d'ailleurs le préfacier du roman de Domi- 
nique Demers, en revendiquant clairement le statut d'écrivain "véritable" dont celle-ci peut se prévaloir avec une ouvre qui lui permet de faire son entrée dans la littérature pour adultes ${ }^{14} \ldots$

La littérature pour la jeunesse ne mériterait donc pas la même attention que les autres genres littéraires, et c'est pourquoi ses écrivains jouiraient d'un statut qui répondrait à ce manque de reconnaissance. D'ailleurs, combien de professeurs de lettres à l'université, d'étudiants universitaires, d'écrivains et d'autres professionnels du livre ont-ils assisté aux colloques en littérature de jeunesse organisés dans le cadre de l'Acfas ou dans d'autres cadres? L'ensemble du champ littéraire et le champ littéraire jeunesse forment deux champs distincts, et les préjugés contre les écrivains jeunesse ne sauraient donc, pour l'essentiel, être fondés que sur l'ignorance de leurs textes.

Au cours des années 1990 , on voit surgir des conflits au sein même du milieu jeunesse, conflits que nous qualifions de "signes du progrès du sous-champ. à cause de la nouveauté et de la multiplicité des positions. Le sous-champ, en quelque sorte, s'enrichit de nouvelles visions. Par exemple, pour les membres de l'Association des écrivaines et des écrivains québécois pour la jeunesse (AEQJ), le métier d'écrivain pour la jeunesse est en péril:

Avec la crise économique et la concurrence féroce qui sévit sur le marché du livre pour la jeunesse, nous risquons, au contraire, d'avoir à affronter des difficultés grandissantes. [...] L'état coupe son aide, les éditeurs négocient les droits d'auteur à la baisse, la liberté d'expression des créateurs est de plus en plus menacée par le mouvement "politiquement correct ", le livre tend à devenir de plus en plus un objet commercial et de moins en moins une ceuvre d'art originale, les conflits d'intérêt se multiplient, les médias continuent de bouder la littérature pour enfants, etc. ${ }^{15}$.

Alors qu'il interroge à nouveau ce thème cle la marginalité, Daniel Mativat, qui a soutenu une thèse à l'Université de Sherbrooke sur le statut socio-économique de l'écrivain au XIX siècle, reconnaît ailleurs le statut secondaire qui échoit à la littérature pour la jeunesse, tout en observant, néanmoins, que

14. Dominique Demers, Marie-Tempête, préface de Jacques Allard, Montréal, Québec/Amérique, 1997, p. 9.

15. Daniel Mativat, "L'A.E.Q.J... Un an déjà!" Le Bulletin, Montréal, n" 4, mai 1993, p. 1. 
[...] l'écrivain-jeunesse, en dépit des multiples contraintes qu'on lui impose pour standardiser son écriture, a toujours le sentiment d'être $[\ldots]$ un écrivain à part entière ${ }^{16}$.

C'est pourquoi le statut d'écrivain à part entière reste toujours l'objet d'une revendication, même au milieu des années 1990, et l'AEQJ milite précisément en ce sens.

Malgré les succès récents de la littérature québécoise pour la jeunesse, il reste encore fort à faire pour parvenir à toute la reconnaissance dont jouit la littérature générale, en rendant notamment la documentation accessible ou encore en lui assurant une couverture médiatique à la mesure de la production et un enseignement génêralisé dans les universités. C'est la raison pour laquelle nous ne pouvons parler que d'une relative autonomie du champ littéraire pour la jeunesse. Si, comme le dit Bourdieu, "c'est la lutte même qui fait l'histoire du champ; c'est par la lutte qu'il se temporalise", on peut dire que le champ littéraire pour la jeunesse a accédé à une relative autonomie au cours des années 1980, après la mise en place et la restructuration des années 1970. L'état de champ séparé s'instaure dans les années 1980 et, désormais, un champ autonome est maintenant capable d'imposer sa logique spécifique.

Quels facteurs ont contribué à unifier et à fortifier le champ jeunesse? L'accélération de la production, le développement de la distribution et de la promotion ont favorisé l'élaboration d'un appareil critique (prix littéraires, critique, information, recherche, enseignement, etc.). Des indices trahissent l'établissement d'un champ spécifique, qu'il s'agisse de la taille du marché (un livre québécois sur trois est actuellement destiné aux jeunes), de l'importance des tirages (deux à trois fois plus élevés que les livres de littérature générale) et de la durée de vie des livres (plusieurs années, alors que les autres livres ne tiennent souvent que quelques mois en librairie).

\section{Définitions de l'écrivain selon Dominique Noguez}

On peut comparer le statut de l'"auteur jeunesse" à celui de l'écrivain reconnu comme tel et, en adoptant le point de vue que

16. Daniel Mativat, "Quelques vacheries", Le Bulletin, Montréal, n" 7, septembre 1994, p. 1 et 5 . 
confère l'autonomisation du champ littéraire jeunesse, apercevoir ce qui les distingue. En revanche, la notion de "grantécrivain" (en un seul mot), introduite et illustrée par Dominique Noguez, convient tout aussi bien à l'un ou l'autre de ces deux types d'écrivain, de sorte qu'elle est même susceptible de nous les faire envisager sous un jour où ce qui les réunit par-delà leurs différences prétendues devient beaucoup plus sensible. Le grantécrivain,

[...] c'est un individu dont la "grandeur" a été reconnue - et non seulement reconnue, mais utilisée et même mythifiée, au sens de Barthes - de son vivant même. Le grantécrivain, c'est cet acteur non seulement de la scène littéraire, mais de la scène culturelle et de la scène publique en général, qui se promène sur terre non seulement - comme tout écrivain - en représentant de commerce de lui-même, mais aussi en incarnation de la conscience universelle ${ }^{17}$.

L' auteur jeunesse" ne peut-il pas à son tour soutenir avantageusement la comparaison avec ce type d'écrivain? N'est-il pas, lui aussi, "un écrivain qui connaît ce luxe, avoir des lecteurs, un auteur qu'on suit, dont on s'arrache l'œuvre nombreuse ${ }^{18}$ "? N'assure-t-il pas, lui aussi, "le service après-vente"? "Interviews, conférences, préfaces ou postfaces aux nouvelles éditions [...], réponse aux lettres de lecteurs ${ }^{19}$. Le grantécrivain dont parle Noguez fait "ouvre d'imagination, de fabulation, de fiction ${ }^{20}$ ". Il est aussi "écrivain dans plusieurs genres ${ }^{21}$. Le grantécrivain est généralement aussi un "écrivain d'idées" (selon l'expression de Julien Benda), qu'il exprime à l'occasion de préfaces, de discours, de lettres ouvertes, mais aussi de textes théoriques, notamment philosophiques ${ }^{22}$. Ces quelques réflexions de Noguez sur les traits et sur les caractéristiques du grantécrivain peuvent s'appliquer dans une large mesure à l'،auteur jeunesse" qui, comme tout écrivain, doit expliquer et défendre ses œuvres.

En conclusion, l'idée d'un sous-champ littéraire québécois pour la jeunesse qui s'est transformé, depuis les années 1980, en champ littéraire propre, introduit une distinction qui porte à

17. Dominique Noguez, Le grantécrivain et autres textes, Paris, Gallimard, coll. "L'infini ", 2000, p. 13.

18. Le grantécrivain, ouvr. cité, p. 17.

19. Le grantêcrivain, ouvr. cité, p. 19.

20. Le grantécrivain, ouvr. cité, p. 16.

21. Le grantécrivain, ouvr. cité, p. 20.

22. Le grantécrivain, ouvr. cité, p. 25. 
croire - ce qui, du reste, n'est pas totalement faux - que la littérature pour la jeunesse est évacuée de la littérature générale, alors qu'elle possède plutôt son propre appareil critique. Une littérature spécialisée, un public spécialisé, des agents spécialisés, avec ses bibliothécaires, ses libraires, ses enseignants, ses animateurs, ses jurés, etc. : tout cela procède d'un lectorat. Les a auteurs jeunesse" n'entrent pas impunément dans le circuit de la littérature générale, qui forme un autre champ, indépendant. Inversement, le succès des "auteurs jeunesse" en indispose plus d'un, car les écrivains pour adultes n'entrent pas comme ils le veulent, eux non plus, dans la sphère jeunesse. En effet, ils ne sont pas très bien accueillis par leurs collègues, ces "auteurs jeunesse", qui leur reprochent de ne pas bien connaître ce type de littérature, et d'être attirés surtout par les tirages plus élevés et par la prétendue facilité de l'entreprise. Il faut dire que plus d'un a vu ses attentes trompées, et pas des moindres. Songeons par exemple à Jacques Godbout et à son Histoire de chasse: à notre connaissance, il n'a pas jugé opportun depuis lors de renouer avec la pratique de la littérature pour la jeunesse.

Que nous réservent les prochaines décennies si les deux sphères s'interpénètrent? La courte échelle, qui a longtemps publié exclusivement des livres pour les jeunes, publie maintenant des romans pour adultes et recherche des écrivains pour adultes dans le but d'alimenter ses collections jeunesse. De plus en plus d'éditeurs tendent à publier leurs romans jeunesse en volumes destinés aux adultes. À long terme, à quelle sphère - à quel champ - tout cela profitera-t-il? 